\title{
How Do Different Anterior Tibial Tendon Transfer Techniques Influence Forefoot and Hindfoot Motion?
}

\author{
A. R. Knutsen MS, T. Avoian MD, S. N. Sangiorgio PhD, S. L. Borkowski MS, \\ E. Ebramzadeh PhD, L. E. Zionts MD
}

Received: 18 July 2014 / Accepted: 10 November 2014/Published online: 25 November 2014

(C) The Association of Bone and Joint Surgeons \& 2014

\begin{abstract}
Background Idiopathic clubfoot correction is commonly performed using the Ponseti method and is widely reported to provide reliable results. However, a relapsed deformity may occur and often is treated in children older than 2.5 years with repeat casting, followed by an anterior tibial tendon transfer. Several techniques have been described, including a whole tendon transfer using a two-incision technique or a three-incision technique, and a split transfer, but little is known regarding the biomechanical effects of these transfers on forefoot and hindfoot motion.

Questions/purpose We used a cadaveric foot model to test the effects of three tibialis anterior tendon transfer techniques on forefoot positioning and production of hindfoot valgus.

Methods Ten fresh-frozen cadaveric lower legs were used. We applied $150 \mathrm{~N}$ tension to the anterior tibial
\end{abstract}

The institution of the authors has received, during the study period, funding from the Doctors' Education Research Fund (J. Vernon Luck, Sr., M.D. Orthopaedic Research Center, Los Angeles, CA, USA). Each author certifies that he or she, or a member of his or her immediate family, has no funding or commercial associations (eg, consultancies, stock ownership, equity interest, patent/licensing arrangements, etc) that might pose a conflict of interest in connection with the submitted article.

Funding for this study was provided by the Doctors' Education Research Fund.

All ICMJE Conflict of Interest Forms for authors and Clinical Orthopaedics and Related Research ${ }^{\circledR}$ editors and board members are on file with the publication and can be viewed on request.

Each author certifies that his or her institution approved or waived approval for the human protocol for this investigation and that all investigations were conducted in conformity with ethical principles of research.

This work was performed at the Orthopaedic Institute for Children, J. Vernon Luck, Sr. M.D. Orthopaedic Research Center, Los Angeles, CA, USA. tendon, causing the ankle to dorsiflex. Three-dimensional motions of the first metatarsal, calcaneus, and talus relative to the tibia were measured in intact specimens, and then repeated after each of the three surgical techniques.

Results Under maximum dorsiflexion, the intact specimens showed $6^{\circ}\left(95 \% \mathrm{CI}, 2.2^{\circ}-9.4^{\circ}\right)$ forefoot supination and less than $3^{\circ}\left(95 \% \mathrm{CI}, 0.4^{\circ}-5.3^{\circ}\right)$ hindfoot valgus motion. All three transfers provided increased forefoot pronation and hindfoot valgus motion compared with intact specimens: the three-incision whole transfer provided $38^{\circ}$ $\left(95 \% \mathrm{CI}, 33^{\circ}-43^{\circ} ; \mathrm{p}<0.01\right)$ forefoot pronation and $10^{\circ}$ (95\% CI, $8.5^{\circ}-12^{\circ} ; \mathrm{p}<0.01$ ) hindfoot valgus; the split transfer, $28^{\circ}\left(95 \% \mathrm{CI}, 24^{\circ}-32^{\circ} ; \mathrm{p}<0.01\right)$ pronation, $9^{\circ}$ (95\% CI, $7.5^{\circ}-11^{\circ} ; \mathrm{p}<0.01$ ) valgus; and the two-incision transfer, $25^{\circ}\left(95 \% \mathrm{CI}, 20^{\circ}-31^{\circ} ; \mathrm{p}<0.01\right)$ pronation, $6^{\circ}$ (95\% CI, $\left.4.2^{\circ}-7.8^{\circ} ; \mathrm{p}<0.01\right)$ valgus.

A. R. Knutsen, S. L. Borkowski

Department of Biomedical Engineering, University of California Los Angeles, Los Angeles, CA, USA

A. R. Knutsen, S. N. Sangiorgio, S. L. Borkowski,

E. Ebramzadeh $(\square)$

The J. Vernon Luck, Sr. M.D. Orthopaedic Research Center, Orthopaedic Institute for Children, 403 West Adams Boulevard,

Los Angeles, CA 90007, USA

e-mail: Edward.Ebramzadeh@ucla.edu

T. Avoian

Orthopaedic Institute for Children, Los Angeles, CA 90007, USA

S. N. Sangiorgio, E. Ebramzadeh, L. E. Zionts

Department of Orthopaedic Surgery, University of California,

Los Angeles, Los Angeles, CA, USA 
Conclusion All three techniques may be useful and deliver varying degrees of increased forefoot pronation, with the three-incision whole transfer providing the most forefoot pronation. Changes in hindfoot motion were small.

Clinical Relevance Our study results show that the amount of forefoot pronation varied for different transfer methods. Supple dynamic forefoot supination may be treated with a whole transfer using a two-incision technique to avoid overcorrection, while a three-incision technique or a split transfer may be useful for more resistant feet. Confirmation of these findings awaits further clinical trials.

\section{Introduction}

Idiopathic clubfoot correction often is achieved using a series of manipulations and cast applications followed by a percutaneous Achilles tenotomy and postcorrective bracing, known as the "Ponseti method" [20]. This technique provides reliable results, however, relapsed deformity has been reported in as many as $54 \%$ of patients [2]. A lateral transfer of the anterior tibial tendon may be performed to correct the dynamic supination of the forefoot that often accompanies a relapse in children older than 2.5 years [3]. As the Ponseti method has become the standard treatment for patients with clubfoot [26], the number of children undergoing an anterior tibial tendon transfer likely will increase. Early recognition and appropriate treatment of relapsed deformity is an important part of the Ponseti method of clubfoot correction [3, 20, 21].

Several techniques have been used to transfer the anterior tibial tendon. In 1940, Garceau [7] described transfer of the whole tendon as a procedure to treat recurrent clubfoot deformity. Garceau rerouted the tendon by pulling it proximally and reinserting it more laterally beneath the extensor retinaculum. This technique used three incisions: one over the anterior aspect of the distal lower leg just above the ankle and two over the dorsum of the foot. He originally anchored the tendon to the base of the fifth metatarsal, but later he and Palmer [8] modified the procedure by attaching the tendon in the cuboid if it was not of sufficient length. More recently, other authors have recommended that the tendon be placed in the third cuneiform to avoid overcorrection of the foot $[5,16,17]$.

Ponseti and Smoley [22] further modified Garceau's technique [7] to use a less-invasive procedure in which the tendon was shifted laterally, distal to the extensor retinaculum, through a subcutaneous tunnel between dorsomedial and dorsolateral foot incisions to the third cuneiform where it was anchored. This modification avoided the need to reroute the tendon proximally, and eliminated the need for a third incision above the ankle. Numerous authors have used this technique and have reported promising clinical results $[6,13,25]$.
Hoffer et al. [10, 11] described a split anterior tibial tendon transfer for treatment of a spastic equinovarus foot in children with cerebral palsy. The procedure is performed through three incisions, with the lateral tendon half rerouted proximal to the flexor retinaculum and anchored in the cuboid. The split anterior tibial tendon transfer was designed to convert the deforming force of a spastic tibialis anterior muscle to a pure dorsiflexor and minimize the risk of overcorrection. The split tendon, having insertions on the medial and lateral sides of the foot, is intended to provide balance so that it neither inverts nor everts the foot $[18,19]$. This transfer also has been used to treat residual dynamic forefoot supination after clubfoot treatment [1, 16, 24].

Few laboratory studies have addressed the biomechanics of the foot after anterior tibial tendon transfers. For instance, plantar pressures [9] and tendon moment arms [19] have been measured to compare the effects of whole and split transfers; however, only one study, to our knowledge, has measured ankle and foot motions as a function of anterior tibial tendon transfer in a cadaver model [12]. Specifically, Hui et al. [12] used electrogoniometers to monitor ankle motion and forefoot motion to determine the optimal site to anchor the anterior tibial tendon and to compare split with whole transfers; however, hindfoot motion was not specifically measured, and the two-incision transfer favored by Ponseti and Smoley was not included [22].

The purpose of our study was to determine the effect of forefoot positioning and hindfoot motion produced by the three anterior tendon transfer techniques using a cadaveric foot model.

\section{Methods}

Ten fresh-frozen cadaveric lower legs, disarticulated at the knee, were used. Each specimen was tested as follows: (1) intact; (2) with the anterior tibial tendon transferred as described by Ponseti and Smoley [22] (two-incision whole transfer); (3) with the anterior tibial tendon transferred as described by Garceau and Palmer [8] modified to insert into the third cuneiform (three-incision whole transfer); and (4) with a split anterior tibial tendon transfer as described by Hoffer et al. [10, 11] (Fig. 1). To minimize variability, all procedures were performed by the same surgeon (TA), a pediatric orthopaedic fellow, under the direction of the senior author (LEZ), a pediatric orthopaedic surgeon. The clinicians played no role in the collection or analysis of the data.

The proximal end of each tibia was secured in a custom aluminum ring using pointed screws, leaving the fibula free of any constraint. The aluminum ring was rigidly attached to the load actuator of a biaxial MTS 858 servohydraulic load frame (MTS Systems Corporation, Minneapolis, MN, 
Fig. 1 A-D Orientation of the anterior tibialis tendon is shown for (A) an intact tendon; (B) with the two-incision anterior tibial tendon transfer, routed distally; (C) with the three-incision anterior tibial tendon transfer, routed proximally; and (D) with a split anterior tibial tendon transfer.

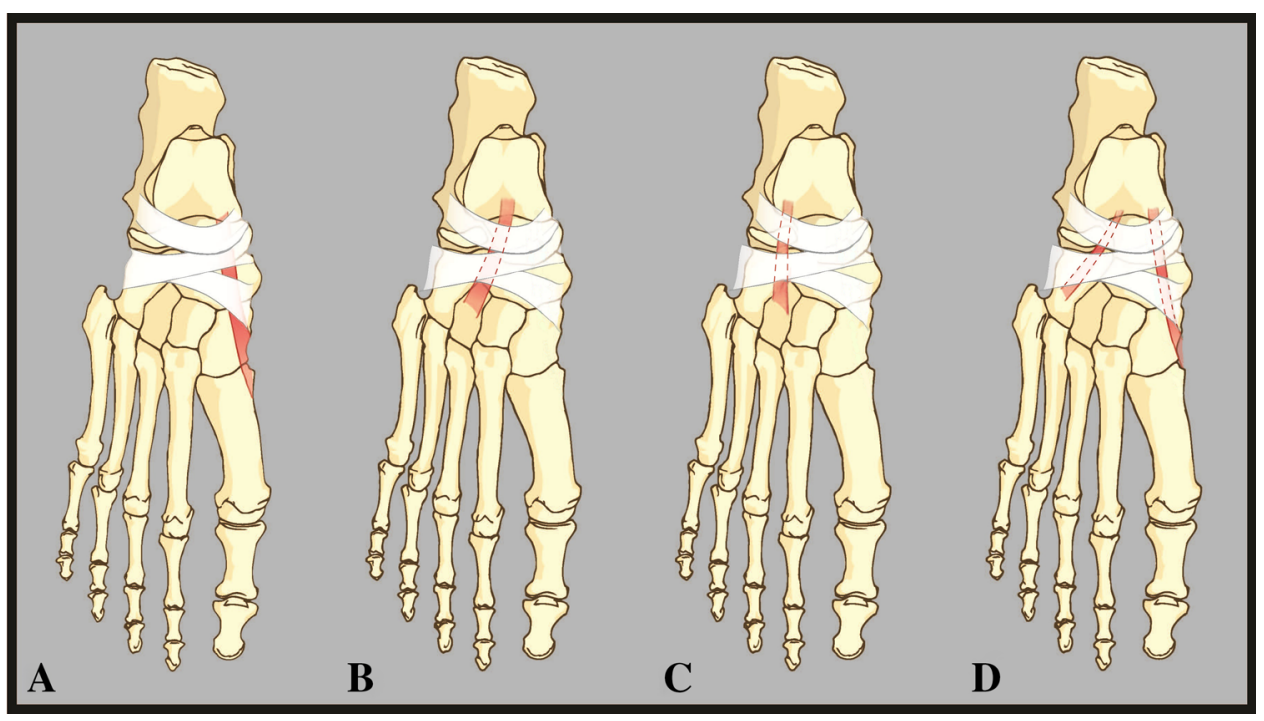

routed through a pulley system attached to an aluminum ring to recreate the natural direction of the muscle forces. The rope then was secured to a load cell which was rigidly attached to the stationary cross-head of the load frame and measured tension in the tendon in real time (Fig. 2).

Motion of each rigid body (described below) was measured throughout experimentation using an Optotrak ${ }^{\circledR}$ 3020 Motion Capture System (Northern Digital Inc, Waterloo, Ontario, Canada). The system uses infrared light-emitting diode (LED) markers creating three-dimensional (3-D) positions, which are captured by three chargecoupled devices paired with three lenses, sequentially, at 3500 samples per second. The software calculates the 3-D position for each marker and displays its coordinates in real time. This motion tracking system has an accuracy of 0.1 $\mathrm{mm}$, a resolution of $0.01 \mathrm{~mm}$ at a distance of $2.25 \mathrm{~m}$, and has been used in previous studies [4, 23]. Motion-tracker flags were created by mounting four LED markers equidistant from the center of a 1.5-inch square plastic fixture, creating a noncollinear 1-inch-square arrangement.

A total of five flags were used. One was mounted on one of the stationary posts of the load frame to be used as a reference. Using cortical bone screws, the remaining four flags were mounted to (1) the medial aspect of the distal tibia; (2) the head of the first metatarsal; (3) the lateral aspect of the calcaneus; and (4) the medial aspect of the talus (Fig. 2). This arrangement allowed us to measure the motions of the first metatarsal, calcaneus, and talus, relative to the tibia.

In addition to motion-tracker flags, a digitizing probe was used to digitize imaginary points on the foot and tibia that tracked motion in reference to each of the flags. Using this system, imaginary points shift relative to the axis of the rigidly fixed motion-tracker flag secured to the bone. Points along the axis of the tibia and on the distal and proximal
USA), such that the long axis of the tibia was aligned with the linear actuator. The distal end of the lower leg was left completely unconstrained. An incision was made in the shaft of the leg to expose the proximal end of the anterior tibial tendon. Near the musculotendinous junction, the tendon was sutured and attached to a rope. The rope was 
talus were used to calculate ankle motion. The projection of the angle between the tibia and talus on the sagittal plate was defined as plantar or dorsal flexion. Points along the axis of the tibia and on the medial and lateral sides of the first metatarsal were used to calculate forefoot motion. The projection of the angle between the long axis of the tibia and first metatarsal on the coronal plane was defined as supination or pronation. Points along the axis of the tibia and the calcaneus were used to calculate hindfoot motion. The projection of the angle between the tibia and calcaneus on the coronal plane was defined as varus or valgus. In addition, points on the medial talus and on the medial and lateral calcaneus were used to calculate hindfoot motion.

Points were digitized relative to the flag on the stationary post of the load frame to create the coronal and sagittal planes, with the long axis of the tibia as the origin. The points on the foot and tibia, described previously, were projected on these planes to calculate joint motions.

The actuator of the load frame initially was positioned so that nearly no tension $(<0.5 \mathrm{~N})$ was exerted on the anterior tibialis tendon. The actuator then was increased at a rate of $1 \mathrm{~mm} / \mathrm{second}$, raising the leg and creating tension in the tendon, causing the foot to dorsiflex. The actuator was stopped when $15^{\circ}$ dorsiflexion or $150 \mathrm{~N}$ tension in the tendon was reached. Each foot was loaded in the same manner first with tendon in the intact position and then again after each of the transfer procedures.

A 6-cm incision was made to expose the tendon at the musculotendinous junction to attach the rope. A second 4$\mathrm{cm}$ incision was made over the dorsomedial aspect of the foot to expose the anterior tibial tendon from just distal to the extensor retinaculum, down to its insertion at the distal part of the first metatarsal and first cuneiform. A third 3-cm incision was made to expose the dorsum of the cuboid, and a fourth 3-cm incision was made to expose the dorsum of the third cuneiform. Cortical screws were then rigidly attached to the first metatarsal base, the center of the cuboid, and the lateral cuneiform for later attachment of the tendon. Accurate placement of the screws was verified using fluoroscopy.

After intact loading was complete, the anterior tibial tendon was detached from its insertion point and a whip suture was applied to the distal end of the tendon. First, we simulated the two-incision transfer technique of Ponseti and Smoley [22]. The whole tendon was rerouted distally beneath the extensor retinaculum by shifting it to the third cuneiform subcutaneously between the dorsal foot incisions. The tendon was attached to the lateral cuneiform cortical screw by the sutures. After loading, the tendon was detached from the lateral cuneiform and the most commonly used technique, the modified three-incision transfer technique of Garceau and Palmer [8] was simulated. The whole tendon was pulled out from under the extensor retinaculum into the proximal skin incision above the ankle. It then was rerouted beneath the extensor retinaculum more laterally and attached to the lateral cuneiform via the cortical screw. After loading, the tendon was detached again and pulled out from under the extensor retinaculum through the proximal skin incision and the split anterior tibial tendon transfer, as described by Hoffer et al. [10, 11], was tested. The tendon was split in half to the proximal extent of the tendon. A whip suture was applied to secure each of the limbs of the split tendon. The lateral half of the tendon then was rerouted under the extensor retinaculum to the cuboid, the medial half was routed to the initial insertion position, and both ends were attached to the cortical screws. The neutral forefoot position was maintained during all tendon transfers and, during the split transfer, care was taken to equalize the tension in both halves of the tendon, as it is attempted during the actual surgery. Loading then was repeated a final time. In this way, each tendon transfer procedure was tested in all 10 specimens, allowing each specimen to serve as its own control.

All of the specimens reached dorsiflexion above the neutral position before the $150 \mathrm{~N}$ tension limit. Two of the 10 specimens reached $15^{\circ}$ dorsiflexion, while the remaining specimens reached an average of $9^{\circ}$ dorsiflexion (range, $1^{\circ}-14^{\circ}$ ).

A power analysis was conducted to determine the minimum sample size required. A SD of $3^{\circ}$, as reported by Hui et al. [12], and a minimum difference of $3^{\circ}$, with a power of $80 \%$ and $\alpha$ of 0.05 , yielded a sample size of 10 .

MATLAB ${ }^{\circledR}$ software (The MathWorks Inc, Natick, MA, USA) was used to calculate angles for ankle motion (plantar and dorsal flexion), forefoot motion (supination and pronation), hindfoot motion (varus and valgus), and subtalar joint motion. SPSS ${ }^{\circledR}$ Statistical Software, Version 15, (IBM, Armonk, NY, USA) was used to perform a paired t-test to compare the three tendon transfer methods at two ankle positions: (1) with the ankle in the neutral position (a $90^{\circ}$ angle between the tibia and foot), and (2) with the ankle in the maximum dorsiflexed position.

\section{Results}

The three transfer procedures each resulted in a different magnitude of forefoot pronation, whether the foot was in neutral or maximum dorsiflexion. These magnitudes of pronation were larger than their corresponding pronation values as measured for the intact specimens (Fig. 3). Specifically, with the ankle in neutral dorsiflexion, the three-incision whole transfer and the split transfer produced greater increases in forefoot pronation than the twoincision transfer $(\mathrm{p}<0.01)$ (Table 1). Likewise, with the ankle in maximum dorsiflexion, the three-incision whole transfer produced a greater increase in forefoot pronation 
Fig. 3 For each of the three procedures, increase in pronation of the forefoot compared with pronation of the corresponding forefoot when measured intact is shown. The increases are shown for neutral dorsiflexion and maximum dorsiflexion. The bars represent the means, with error bars representing SD.

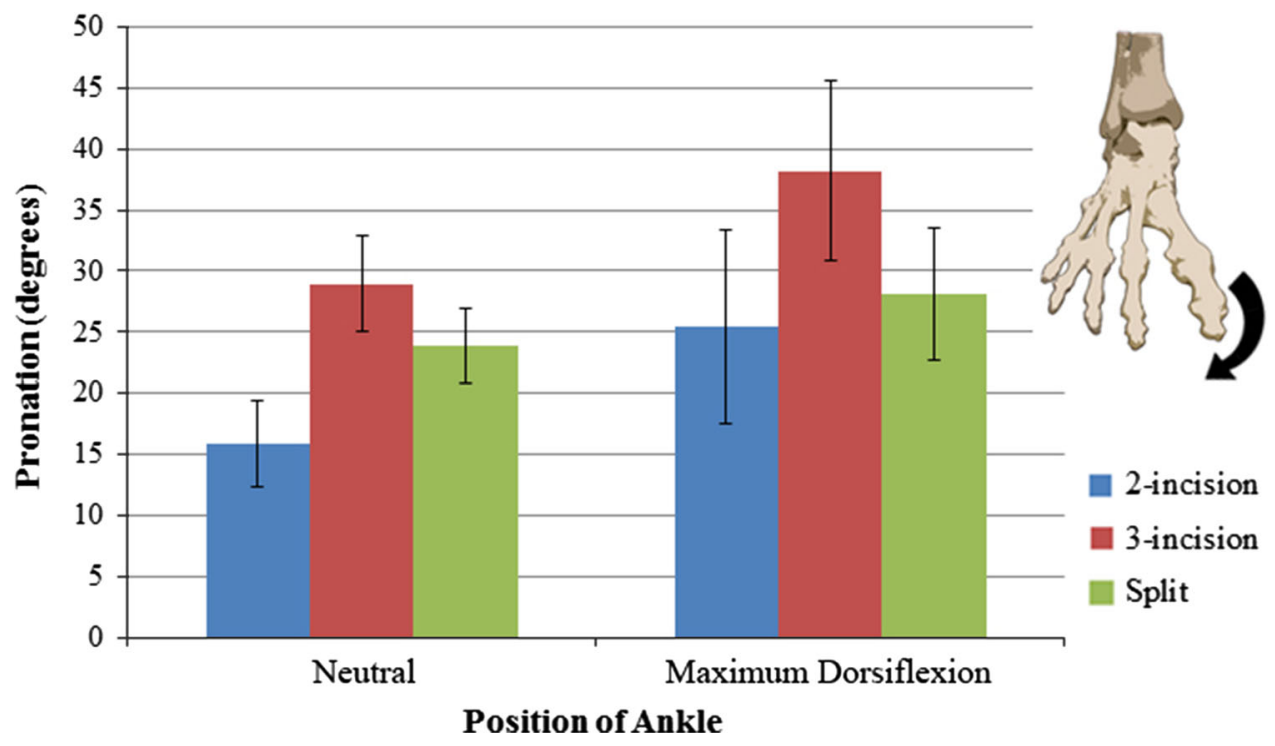

Table 1. Comparison of tendon transfer procedures

\begin{tabular}{|c|c|c|c|c|}
\hline \multirow[t]{2}{*}{ Procedures } & \multicolumn{2}{|l|}{ Neutral } & \multicolumn{2}{|l|}{ Maximum dorsiflexion } \\
\hline & Mean \pm SD $(95 \%$ CI $)$ & $\mathrm{p}$ value & Mean \pm SD $(95 \%$ CI $)$ & $\mathrm{p}$ value \\
\hline \multicolumn{5}{|l|}{ Forefoot motion } \\
\hline Two-incision whole transfer versus & $16 \pm 3.5(13-18)$ & & $25 \pm 7.9(20-31)$ & \\
\hline Three-incision whole transfer & $29 \pm 3.9(26-32)$ & $<0.01$ & $38 \pm 7.3(33-43)$ & $<0.01$ \\
\hline Split transfer & $24 \pm 3.1(22-26)$ & $<0.01$ & $28 \pm 5.4(24-32)$ & 0.24 \\
\hline \multicolumn{5}{|l|}{ Three-incision whole transfer versus } \\
\hline Split transfer & & $<0.01$ & & $<0.01$ \\
\hline \multicolumn{5}{|l|}{ Hindfoot motion } \\
\hline Two-incision whole transfer versus & $3.5 \pm 2.1(2.0-5.0)$ & & $6.0 \pm 2.5(4.2-7.8)$ & \\
\hline 3-incision whole transfer & $9.5 \pm 3.5(7.0-12)$ & $<0.01$ & $10 \pm 2.4(8.5-12)$ & $<0.01$ \\
\hline Split transfer & $8.0 \pm 2.9(5.9-10)$ & $<0.01$ & $9.4 \pm 2.7(7.5-11)$ & $<0.01$ \\
\hline \multicolumn{5}{|l|}{ Three-incision whole transfer versus } \\
\hline Split transfer & & 0.17 & & 0.06 \\
\hline
\end{tabular}

than the two-incision whole transfer or the split transfer ( $\mathrm{p}$ $<0.01$ ) (Table 1).

The three transfer procedures resulted in varying magnitudes of hindfoot valgus, regardless whether the foot was in neutral or maximum dorsiflexion. These magnitudes of hindfoot valgus were, on average, larger than their corresponding valgus values as measured for the intact specimens (Fig. 4). Specifically, with the ankle in neutral dorsiflexion, the three-incision and the split transfer procedures produced larger increases in valgus motion than the two-incision transfer $(\mathrm{p}<0.01)$ (Table 1). Similarly, with the ankle in maximum dorsiflexion, the three-incision whole transfer and the split transfer produced larger increases in valgus motion than the two-incision transfer $(\mathrm{p}<0.01)$ (Table 1).

\section{Discussion}

After successful treatment of a clubfoot deformity by the Ponseti method, a relapse may occur, and an important component of treating a relapsed deformity includes a lateral transfer of the anterior tibial tendon. The presentation of a relapsed clubfoot is variable with some patients having only dynamic supination of the forefoot, and others with varying degrees of deformity owing to mild residual joint malalignment $[3,20]$, or marked weakness of the peroneal musculature $[5,7,16,25]$.

Because an anterior tibial tendon transfer addresses the dynamic component of a relapsed clubfoot, sufficient correction of any fixed deformity needs to be achieved before tendon transfer. For many younger patients who experience 
Fig. 4 For each of the three procedures, increase in valgus motion of the hindfoot compared with valgus motion of the corresponding hindfoot when measured intact is shown. The increases are shown for neutral dorsiflexion and maximum dorsiflexion. The bars represent the means, with error bars representing SD.

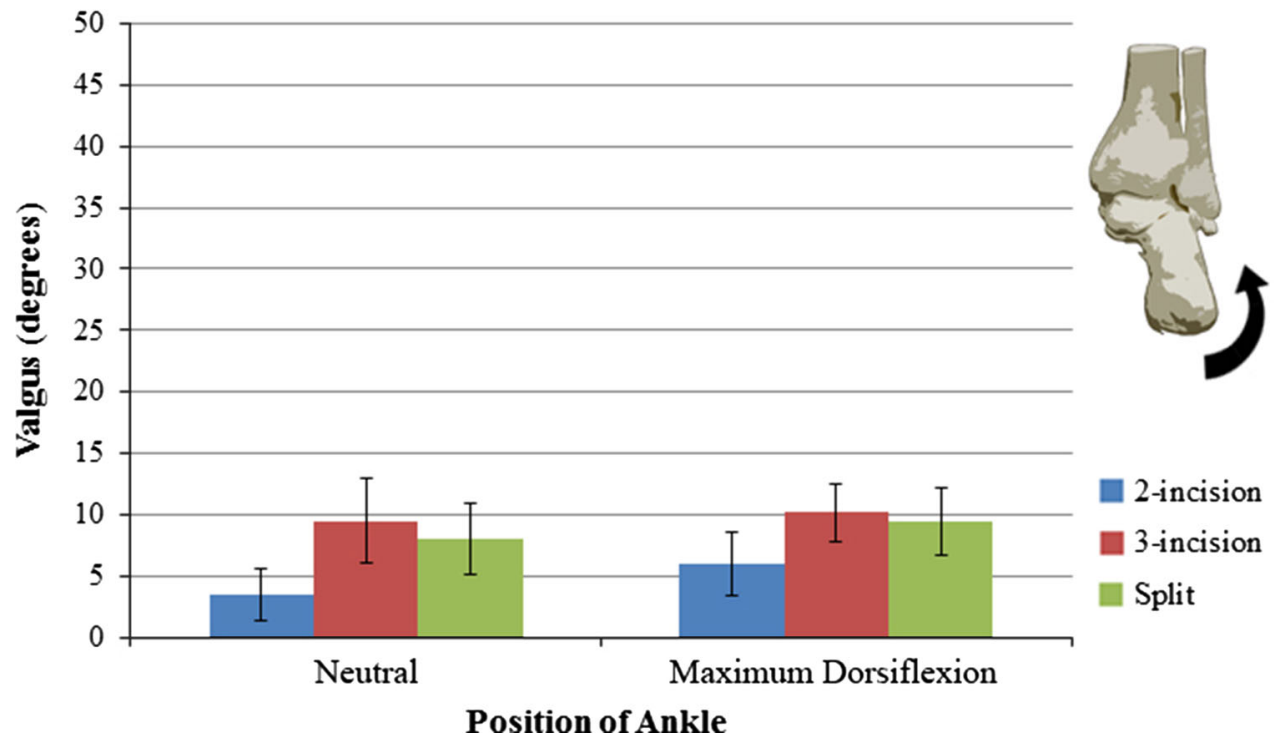

a relapse after treatment using the Ponseti method, sufficient correction of the foot may be achieved with a series of manipulations and casts. If the ankle equinus cannot be adequately addressed with cast treatment alone, heel cord lengthening, or less commonly, a posterior soft tissue release should be done at the time of the transfer. Adequate deformity correction usually cannot be achieved in older patients with residual osseous deformity by cast treatment alone, and these patients require corrective osteotomy so that the transfer may function properly. Several different techniques of anterior tibial tendon transfer have been described [5, 7, 8, 10, 11, 16, 17, 22], but little is known about the effect of those transfers on forefoot position and hindfoot motion. We used a cadaveric foot model to test the effect of three different anterior tibial tendon transfer techniques on forefoot positioning and hindfoot motion. We hoped to learn how the varying techniques altered motion of the foot, which potentially could influence which method might be applicable in different clinical situations.

Our study had several limitations. First, the cadaveric specimens we used were adult lower legs, as pediatric specimens are unavailable in the United States for biomechanics research; therefore, less joint mobility was present than that in children. Additionally, to our knowledge, none of the specimens had osseous deformity or joint malalignment that may be present in children with relapsed clubfeet. As a result, the effect of the transfer in the cadaver specimens may not precisely reproduce the effect that may be seen in the clinical situation. However, adult specimens have been used in place of pediatric specimens in several published studies and have provided meaningful results [12, 15, 23]. Second, anatomic positioning of each specimen had to be approximated, and to minimize variability in the data, the same positioning method was used for each trial. Third, by isolating the effects of the anterior tibialis muscle, other muscles that potentially may influence joint motion were not simulated, and therefore, physiologic loading of the foot was not reproduced. Finally, adaptive changes that may occur in a transferred muscle in living subjects could not be simulated.

The findings from our study suggest that all three of the tendon transfer techniques provided an increase in pronation of the forefoot, with a maximum difference among them of $13^{\circ}$ (Fig. 3). Compared with the intact specimens, the threeincision whole transfer provided $38^{\circ}$ forefoot pronation compared with $28^{\circ}$ for the split transfer and $25^{\circ}$ for the twoincision whole transfer. As the distal anchor point is the same in the two-incision and three-incision whole tendon transfer techniques, perhaps the difference in the amount of pronation produced is attributable to the manner in which the tendon is transferred. Specifically, in the two-incision approach, the tendon is transferred through a subcutaneous tunnel that potentially could weaken the effect of the transfer by providing a somewhat less direct line of pull compared with the three-incision method.

Although there were differences in hindfoot motion among the tendon transfer techniques, these variations were small. Compared with the intact specimens, the three-incision whole transfer produced $10^{\circ}$ valgus compared with $9^{\circ}$ for the split transfer, and $6^{\circ}$ for the two-incision whole transfer. In this study, hindfoot motion was defined as varus and valgus motion between the tibia and the calcaneus from a fixed coronal plane along the tibia. The split transfer was found to produce hindfoot motion, a finding that generally was consistent with previous biomechanical studies [14, 18, 19]. Specifically, previous studies measured the moment arm of the tibialis anterior muscle in anatomic specimens [14], before and after split transfer of the anterior tibial tendon [18, 19]. Moment arms were measured with the talocrural joint fixed in either the neutral position or in plantar flexion by 
securing the tibia to the talus. It was found that, in intact specimens, shortening the tibialis anterior muscle created an inversion moment arm at the subtalar joint [14, 18, 19]. When a split anterior tibial tendon transfer was tested, all specimens had an eversion moment arm. In contrast to previously reported biomechanical studies [14, 18], we found the split anterior tibial tendon transfer resulted in forefoot pronation and hindfoot valgus comparable to that provided by a whole transfer. Hui et al. [12] performed split transfers of the anterior tibial tendon in cadaver specimens routed to different insertion sites. They reported that a split anterior tibial tendon transfer maximized dorsiflexion, while minimizing supination and pronation, consistent with the intended clinical outcome of the procedure described by Hoffer et al. [10, 11].

In our study, each of the three anterior tibial tendon transfer techniques showed different correction potential and therefore may be applicable in different clinical situations, as each patient with relapse is unique. Although applying the outcomes of an adult cadaver model to a dynamic deformity in children who have greater biological plasticity is difficult, the results of our study may be interpreted to suggest that a child with supple dynamic forefoot supination may be best treated using the twoincision technique favored by Ponseti and Smoley [22], because it is less invasive and less prone to overcorrection. However, the three-incision technique proposed by Garceau [7] and Garceau and Palmer [8] or the split transfer described by Hoffer et al. $[10,11]$ may be more applicable for patients who have less soft tissue elasticity or more marked weakness of the peroneal musculature because these procedures provide greater forefoot pronation forces. Confirmation of the usefulness of the different transfer techniques in patients with varying clinical presentations of relapsed clubfoot must await future clinical trials.

\section{References}

1. Asperheim MS, Moore C, Carroll NC, Dias L. Evaluation of residual clubfoot deformities using gait analysis. J Pediatr Orthop B. 1995;4:49-54.

2. Cooper DM, Dietz FR. Treatment of idiopathic clubfoot: a thirtyyear follow-up note. J Bone Joint Surg Am. 1995;77:1477-1489.

3. Dietz FR. Treatment of a recurrent clubfoot deformity after initial correction with the Ponseti technique. Instr Course Lect. 2006; 55:625-629.

4. Ebramzadeh E, Knutsen AR, Sangiorgio SN, Brambila M, Harris TG. Biomechanical comparison of syndesmotic injury fixation methods using a cadaveric model. Foot Ankle Int. 2013;34: 1710-1717.

5. Ezra E, Hayek S, Gilai AN, Khermosh O, Wientroub S. Tibialis anterior tendon transfer for residual dynamic supination deformity in treated club feet. J Pediatr Orthop B. 2000;9:207-211.
6. Farsetti P, Caterini R, Mancini F, Potenza V, Ippolito E. Anterior tibial tendon transfer in relapsing congenital clubfoot: long-term follow-up study of two series treated with a different protocol. $J$ Pediatr Orthop. 2006;26:83-90.

7. Garceau GJ. Anterior tibial tendon transposition in recurrent congenital club-foot. J Bone Joint Surg Am. 1940;22:932-936.

8. Garceau GJ, Palmer RM. Transfer of the anterior tibial tendon for recurrent club foot: a long-term follow-up. J Bone Joint Surg Am. 1967;49:207-231.

9. Henderson CP, Parks BG, Guyton GP. Lateral and medial plantar pressures after split versus whole anterior tibialis tendon transfer. Foot Ankle Int. 2008;29:1038-1041.

10. Hoffer MM, Barakat G, Koffman M. 10-year follow-up of split anterior tibial tendon transfer in cerebral palsied patients with spastic equinovarus deformity. J Pediatr Orthop. 1985;5:432434.

11. Hoffer MM, Reiswig JA, Garrett AM, Perry J. The split anterior tibial tendon transfer in the treatment of spastic varus hindfoot of childhood. Orthop Clin North Am. 1974;5:31-38.

12. Hui JH, Goh JC, Lee EH. Biomechanical study of tibialis anterior tendon transfer. Clin Orthop Relat Res. 1998;349:249-255.

13. Ippolito E, Ricciardi-Pollini PT, Tudisco C, Ronconi P. The treatment of relapsing clubfoot by tibialis anterior transfer underneath the extensor retinaculum. Ital J Orthop Traumatol. 1985;11:171-177.

14. Klein P, Mattys S, Rooze M. Moment arm length variations of selected muscles acting on talocrural and subtalar joints during movement: an in vitro study. J Biomech. 1996;29:21-30.

15. Knutsen A, Avoian T, Borkowski SL, Ebramzadeh E, Zionts LE, Sangiorgio SN. Accuracy of radiographs in assessment of displacement in lateral humeral condyle fractures. J Child Orthop. 2014;8:83-89.

16. Kuo KN, Hennigan SP, Hastings ME. Anterior tibial tendon transfer in residual dynamic clubfoot deformity. J Pediatr Orthop. 2001;21:35-41.

17. Lampasi M, Bettuzzi C, Palmonari M, Donzelli O. Transfer of the tendon of tibialis anterior in relapsed congenital clubfoot: longterm results in 38 feet. J Bone Joint Surg Br. 2010;92:277-283.

18. Piazza SJ, Adamson RL, Moran MF, Sanders JO, Sharkey NA. Effects of tensioning errors in split transfers of tibialis anterior and posterior tendons. J Bone Joint Surg Am. 2003;85:858-865.

19. Piazza SJ, Adamson RL, Sanders JO, Sharkey NA. Changes in muscle moment arms following split tendon transfer of tibialis anterior and tibialis posterior. Gait Posture. 2001;14:271-278.

20. Ponseti IV. Congenital Clubfoot: Fundamentals of Treatment. NY, NY: Oxford University Press; 1996.

21. Ponseti IV. Relapsing clubfoot: causes, prevention, and treatment. Iowa Orthop J. 2002;22:55-56.

22. Ponseti IV, Smoley EN. Congenital club foot: the results of treatment. J Bone Joint Surg Am. 1963;45:261-344.

23. Sangiorgio SN, Borkowski SL, Bowen RE, Scaduto AA, Frost NL, Ebramzadeh E. Quantification of increase in three-dimensional spine flexibility following sequential Ponte osteotomies in a cadaveric model. Spine Deformity. 2013;1:171-178.

24. Sankar WN, Rethlefsen SA, Weiss J, Kay RM. The recurrent clubfoot: can gait analysis help us make better preoperative decisions? Clin Orthop Relat Res. 2009;467:1214-1222.

25. Thompson GH, Hoyen HA, Barthel T. Tibialis anterior tendon transfer after clubfoot surgery. Clin Orthop Relat Res. 2009; 467:1306-1313.

26. Zionts LE, Sangiorgio SN, Ebramzadeh E, Morcuende JA. The current management of idiopathic clubfoot revisited: results of a survey of the POSNA membership. J Pediatr Orthop. 2012;32: $515-520$. 